\title{
Patella Cubiti: Rare Anatomical Variation Case Report and Review of the Literature \\ M Ouali Idrissi ${ }^{1}$, F. Zahra Abakka ${ }^{1 *}$, Y. Zouine ${ }^{1}$, B. Boutakioute ${ }^{1}$, N. CherifIdrissi El Ganouni ${ }^{1}$
}

${ }^{1}$ Radiology AR-Razidepartment, CHU Mohammed VI, University Cadi Ayad, Marrakech, Marocco

DOI: $10.36347 /$ simcr.2021.v09i04.031

| Received: 16.03.2021 | Accepted: 22.04.2021 | Published: 29.04.2021

*Corresponding author: F. Zahra Abakka

Abstract

Case Report

The Cubiti patella is a very rare anomaly characterized by the non-union of the olecranon to the proximal ulna. We report the case of a 45-year-old patient admitted with chronic right elbow pain and no known history of trauma. The diagnosis revealed a patella Cubiti on standard radiography, which was mistaken for an olecranon fracture.

Keywords: Elbow, olecranon, patella.

Copyright (9) 2021 The Author(s): This is an open-access article distributed under the terms of the Creative Commons Attribution 4.0 International License (CC BY-NC 4.0) which permits unrestricted use, distribution, and reproduction in any medium for non-commercial use provided the original author and source are credited.

\section{CASE RePORT}

This is a 45-year-old man, a professional driver with no previous history of trauma, was admitted to our clinic with chronic right elbow pain.

On inspection, there was no swelling opposite the area of pain projection. Palpation revealed a firm painful mass on the posterior aspect of the right elbow, opposite the olecranon, and the patient reported a cracking sound at this level. This symptomatic mass had not been explored before.

A frontal and lateral X-ray of the right elbow was taken, showing a well-corticalized bone-segment opposite the olecranon, with clean, soft edges and no other obvious abnormalities (Fig-1), with an appearance similar to the patella.
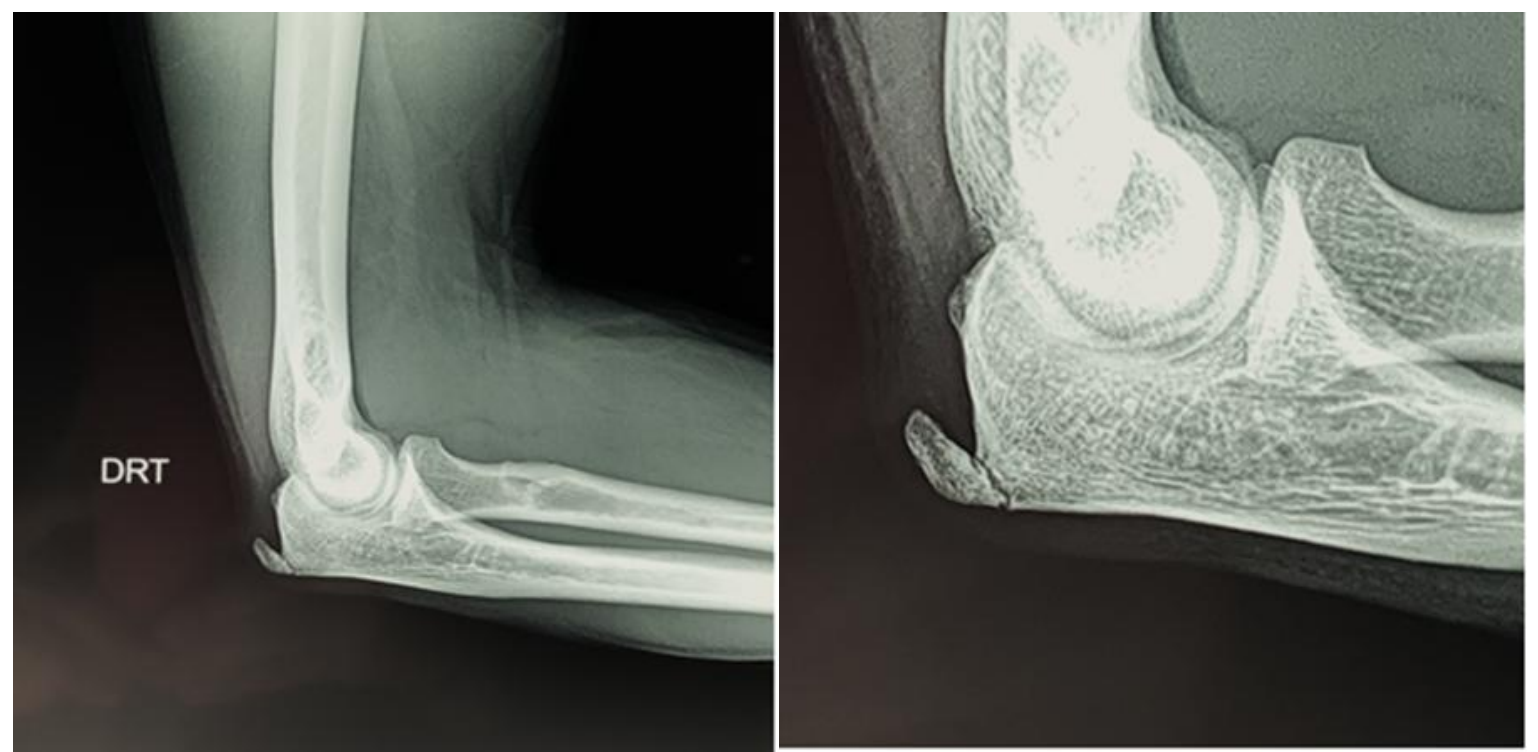

Fig-1: Standard lateral radiograph of the right elbow: Well corticalized calcification, with clear edges and blunt without another anomaly of the elbow

The ultrasound showed calcification next to the enthesis of the tendon of the triceps brachialis muscle (Fig-2). 


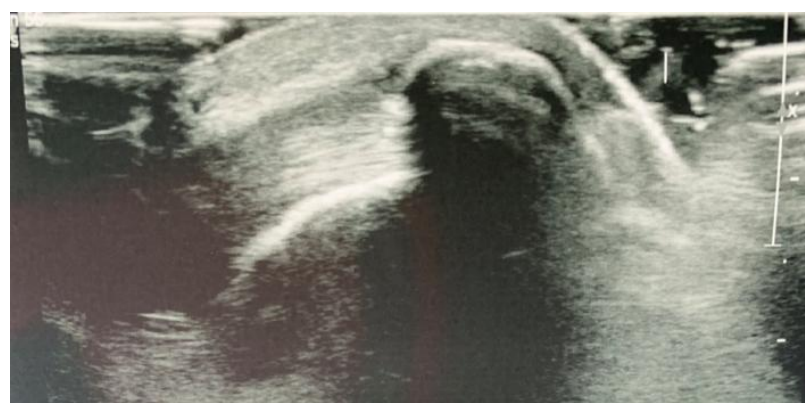

Fig-2: Ultrasound of the right elbow: calcification (arrow) next to the enthesis of the tendon of the Triceps brachialis muscle

\section{DiscuSSION}

Patella Cubiti is a very rare anatomical variation which can be unilateral or bilateral [1]. It corresponds to a sesamoid bone in the tendon of the triceps brachii. In a typical case, the entire olecranon or part of it is separated from the proximal ulna, its bony edges are regular and smooth with a cortical contour on each side [2-4].

Its etiology is unknown, several theories have been proposed: congenital, developmental or traumatic [5]. Kattan has supported a congenital origin as a sesamoid in the tendon of the triceps [3]. Habbe suggested avulsion of the olecranon epiphysis with periosteal stripping of the proximal ulnar shaft [1], while other developmental theories have shown that separation of the epiphysis from the center of the olecranon in early childhood led the independent development of this center [6].

Its discovery is made in the course of traumatic consequences or more often fortuitously on an X-ray of the elbow. It can be asymptomatic or cause symptoms, including limitation of movement, stiffness or pain [7].

The standard X-ray of the front and lateral elbow is performed as a first-line and shows a well corticalized calcification, with clear edges and blunt without any other obvious anomaly (Fig-1) presenting a patella-like appearance. The ultrasound shows a bone fragment next to the enthesis of the tendon of the triceps brachialis muscle.
The differential diagnosis must be made with the post-traumatic non-union of an old fracture of the tip of the olecranon. The Cubiti patella is well demarcated with a smooth and intact cortical surface of the olecranon, while non-union of old fractures should present an irregular surface with a sclera margin of the parent olecranon and distal fracture fragments. Other differential diagnoses include the unfused olecranon epiphysis in young patients and hydroxyapatite deposits in the triceps tendon [5].

Its treatment remains controversial; surgical intervention may be indicated in patients with stiff elbow [7]. Intraoperatively, it is observed that the triceps tendon is intact with individualization of a rounded bone fragment with regular contours at $3 \mathrm{~cm}$ from the beak of the olecranon embedded in this tendon reminiscent of the anatomy of the patella [8].

\section{RÉFÉRENCE}

1. Habbe JE. Patella Cubiti: report of 4 cases. AJR Am J Roentgenol. 1942; 48:513-26.

2. Gunn G. Patella Cubiti. Br J Surg. 1928;15(60):612-5.

3. Kattan K, Babcock DS. Bilateral Patella CubitiCase-Report 105. Skeletal Radiology. 1979 Jan $1 ; 4(4): 249-50$.

4. Van Demark RE, Anderson TR. Fractured patella Cubiti: report of a case with pathologic findings. Clin Orthop Relat Res. 1967; 53:131-134.

5. Khomarwut K, Sutthisast W, Vasuntaraporn U, Arpornchayanon O. Bilateral patellar CUBITI: a case report. The Bangkok Medical Journal. 2019 Feb 20;15(1):91.

6. Thijn CJ, van Ouwerkerk WP, Scheele PM, de Jongh HJ. Unilateral patella cubiti: a probable posttraumatic disorder. European journal of radiology. 1992 Jan 1;14(1):60-2.

7. Mittal R, Sampath Kumar V, Gupta T. Patella Cubiti: a case report and literature review. Arch Orthop Trauma Surg. 2014;134(4):467-71.

8. Mokoko-Louckou AE, Bouhelo-Pam KP, Chaibou B, Abdouli I, Shimi M, Ibrahim AE, Mrini AE. Patella cubiti detected after a trauma: about a case and literature review. The Pan African Medical Journal. 2019 Mar 18:32:128. 